\title{
Caracterización de los macroprocesos de gestión humana para las organizaciones intensivas en conocimiento: análisis de los centros de investigación de excelencia en Colombia
}

Carlos Hernán Gonzalez-Campo

Universidad del Valle Colombia

Colombia

Guillermo Murillo-Vargas

Universidad del Valle Colombia

Colombia

Mónica García- Solarte

Universidad del Valle Colombia

Colombia 


\title{
Caracterización de los macroprocesos de gestión humana para las organizaciones intensivas en conocimiento: análisis de los centros de investigación de excelencia en Colombia
}

\author{
Carlos Hernán Gonzalez-Campo ${ }^{\circledR}$, Guillermo Murillo-Vargas i \& Mónica García- \\ Solarte \\ Universidad del Valle - Colombia
}

Para citaciones: Gonzalez campo, C., Murillo Vargas, G., \& García Solarte, M. (2021). Caracterización de los macroprocesos de gestión humana para las organizaciones intensivas en conocimiento: análisis de los centros de investigación de excelencia en Colombia. Panorama Económico, 29(1), 5-23.

Recibido: 4 de junio de 2020

Aprobado: 25 de noviembre de 2020

Autor de correspondencia:

Carlos Hernan Gonzalez-Campo

carlosh.gonzalez@.correounivalle.edu.co

Editor: Andrés Escobar E. Universidad de Cartagena-Colombia.

Tipología IBN Publindex:

Artículo Resultado de Investigación

Científica y Tecnológica

Copyright: (C) 2021. Gonzalez campo, C., Murillo Vargas, G., \& García Solarte, M. Este es un artículo de acceso abierto, distribuido bajo los términos de la licencia https://creativecommons.org/licenses/by-

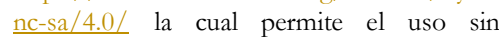
restricciones, distribución y reproducción en cualquier medio, siempre y cuando que el original, el autor y la fuente sean acreditados.

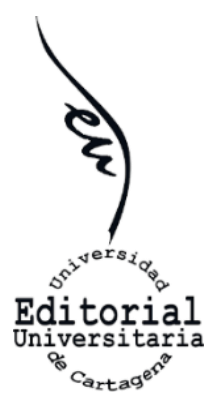

\section{RESUMEN}

En el desarrollo de un país, hoy en día se deben tener en cuenta las Organizaciones Intensivas en Conocimiento (OIC) y el aporte que ellas hacen a los procesos de innovación. En el caso de Colombia, los Centro de Investigación de Excelencia (CIE) son reconocidos por el Sistema Nacional Ciencia, Tecnología e Innovación, desde hace varios años, por sus aportes a la generación de conocimiento de frontera en diferentes áreas. En este contexto, este artículo tiene como objetivo proponer una caracterización de los macroprocesos de gestión humana para OIC, partiendo de una investigación cualitativa que, desde el estudio del caso de los CIE, y específicamente desde el análisis de sus procesos de gestión humana. Es importante reconocer las diferencias existentes entre las organizaciones tradicionales y las OIC, lo que ha implicado entre otros, una revaloración del trabajador y de su rol dentro de cada organización, razón por la cual no pueden ser los mismo la gestión humana dentro de las OIC, esta es una de las conclusiones que se derivan del estudio de los CIE y del contraste de los resultados con la literatura existente, que definen el aporte de este artículo.

Palabras clave: Gestión Humana, Organizaciones Intensivas de Conocimiento, Centros de Investigación de Excelencia.

\section{Characterization of the macro-processes of human management for knowledge-intensive organizations: analysis of the research centers of excellence in Colombia}

\section{ABSTRACT}

Nowadays, Knowledge Intensive Organizations (OIC in Spanish) and the contribution they make to innovation processes must be taken into account for the development of a country. In the case of Colombia, Research Centers of Excellence (CIE in Spanish) have been recognized by the National Science, Technology and Innovation System for several years due to their contributions to the generation of cutting-edge knowledge in different areas. In this context, this article aims to propose a characterization of human management macro-processes for OICS reached from a qualitative research, case-study of the $\mathrm{CIE}$, and specifically from the analysis of their human management processes. It is important to recognize the differences between traditional organizations and OICS which has implied a re-evaluation of the worker and its role within the organization, reason why human management within ICOs cannot be the same. This is one of the conclusions derived from the study of the CIE and from the comparison of the results with previous literature, which defines the contribution of this article. 
Keywords: Human Management, Knowledge-Intensive Organizations, Research Canters of Excellence.

\section{INTRODUCCIÓN}

Hoy en las organizaciones los activos intangibles como el capital humano son importantes al estudiar la gestión de las organizaciones, debido a que son los que poseen conocimiento y dan evidencia de los procesos de innovación de estas, convirtiéndolos en activos valiosos en los procesos administrativos administración (Drucker, 2002; Greenwood, 2009; Krüger, 2006; Lundvall \& Borrás, 1997; Makani \& Marche, 2010; Tapscott, 1997). Por lo tanto, el conocimiento y la innovación hoy pueden asegurar el éxito y sostenibilidad de las organizaciones (Drucker, 2003; Krüger, 2006; Lundvall, 1999; Makani \& Marche, 2010; North \& Rivas, 2008; Schnarch, 2009).

El conocimiento es relevante e importante para las Organizaciones Intensivas en Conocimiento (OIC) y se convierte en factor fundamental en la generación de ventaja competitiva (Swart \& Kinnie, 2003; Makani \& Marche, 2010). Consecuentemente, los trabajadores son el activo relevante, ya que son los que poseen el conocimiento y este el principal elemento para el desarrollo de las OIC (González et al., 2016), Alvesson, 2001; 2004; Makani \& Marche, 2010; Sokeila, Mäkinen \& Näsi, 2003; Starbuck, 1992).

La Gestión Humana, en los procesos administrativos de la organización se convierte en un eje importante, debido a su relación con el capital humano. Para García (2008), la gestión humana hace referencia a todos los procesos y actividades estratégicas de orientación, soporte y apoyo a la Dirección organizacional, la conforman una serie de planeaciones, actividades, programas y políticas, cuyo fin es reclutar, seleccionar, formar, desarrollar, motivar y retribuir personal para potencializar la organización.

El concepto de OIC, según Swart y Kinnie (2003), debe ser asignado únicamente a las organizaciones que, por medio de la explotación del conocimiento tácito, crean valor en el mercado, por medio de la gestión eficaz de la mano de obra altamente calificada (Nonaka y Takeuchi, 1995). Es por ello que esta consideración sobre el capital humano y social innato de las OIC plantea retos para las actividades de gestión humana, principalmente en lo que respecta a la adquisición y el mantenimiento de los trabajadores del conocimiento cualificado y el apoyo a la explotación del conocimiento (Boxall \& Purcell, 2003). Pensar en la gestión humana en las OIC, requiere de nuevos enfoques distintos a los que tradicionalmente han servido a las organizaciones industriales y de servicios, para centrarse en el conocimiento de los empleados y su gestión.

El presente artículo es producto del proyecto de investigación denominado "Generación de un Modelo de Gestión para las Organizaciones Intensivas en Conocimiento", realizado por el grupo de investigación Humanismo y Gestión de la Universidad del Valle. Está compuesto primero por una discusión teórica del concepto de Gestión Humana, como estado del arte se presentan los hallazgos bibliográficos relacionados con los procesos en organizaciones intensivas de conocimiento. Luego se presenta la metodología de la investigación. En la siguiente sección se presentan los hallazgos de la investigación, en esta, a través de una investigación tipo documental y por medio de entrevistas 
estructuradas analizadas con métodos cualitativos se estudian las actividades que realizan los centros de excelencia de investigación en Colombia en materia de gestión humana. Finalmente, se presenta una propuesta de procesos en Gestión Humana para Organizaciones Intensivas de Conocimiento.

\section{MARCO TEÓRICO}

La gestión humana o del talento humano, abarca un grupo de actividades orientadas a influir en el mejoramiento de la relación organización-empleado o, como un conjunto de actividades que según García, Murillo y González (2011) pueden definirse en cinco macroprocesos que son: (1) Organización y Planificación del Área de Gestión Humana, (2) Incorporación y Adaptación de las Personas a la Organización, (3) Compensación, Bienestar y Salud de las Personas, (4) Desarrollo de Personas y (5) Relaciones con el Empleado.

Los macroprocesos de gestión humana buscan generar dentro de las organizaciones cambios orientados al mejoramiento de estas en temas como la elección, el desarrollo y la correcta ubicación de los colaboradores, con el fin de que se sientan motivados con su trabajo y su respuesta sea generar mejor rendimiento, que reditúa en favor de la organización y del mismo colaborador (Decenzo \& Robbins, 2001; Dessler, 1991; García, 2008; Sherman, Bohlander \& Snell, 1999). Las actividades desplegadas a través de la gestión del talento humano deben proporcionar políticas de desarrollo, seguridad, compensación y bienestar en el lugar de trabajo, fortaleciendo el compromiso de los colaboradores en función de la sostenibilidad de las organizaciones.

El desarrollo humano es un elemento primordial que debe ser atendido desde las áreas de gestión humana, en tanto el éxito funcional y la gestión del cambio que tiene lugar en la esfera organizacional depende mayoritariamente de las habilidades y conocimientos que posee el factor humano. Es importante desarrollar el liderazgo, las habilidades humanas y gestionar adecuadamente los equipos de trabajo para que las organizaciones sean sostenibles a lo largo de la cadena de valor, y así, alcancen un rendimiento elevado (Thakur \& Kumar, 2019).

Para García et al. (2011), las organizaciones, a través de una buena gestión de los recursos comprendida como la forma en que los selecciona, en que son asignados, el manejo y lo productivos que resultan, construye su ventaja competitiva. Es así como resulta sensato afirmar, que la correcta gestión de los recursos humanos genera una ventaja competitiva para las organizaciones. Esto requiere que se hagan esfuerzos para llevar a cabo actividades, dedicar recursos económicos y de tiempo para la formación, que permita a los colaboradores un adecuado desarrollo de sus funciones en tanto generan valor en su trabajo. De acuerdo con las capacidades de la organización, se espera también que dichos esfuerzos contribuyan a la satisfacción laboral y la productividad. Atendiendo a estas consideraciones, Montoya, Boyero, y Guzmán (2016) sugieren que en la actualidad ha surgido la necesidad de gestionar el cambio en términos de la misión y la visión organizacional, con el fin de darle mayor protagonismo al factor humano en el proceso de cumplimiento de las propuestas estratégicas. En ese sentido, los colaboradores poseen un rol esencial dentro de los procesos de planeación, ejecución y control, donde la agrupación entre las competencias individuales y grupales que se derivan de ellos, 
garantizan el logro de las proyecciones empresariales a partir de su adaptación a los retos de la globalización e internacionalización.

De acuerdo con Pérez y Lopera (2016) la gestión humana y sus acciones globales si bien son objeto de medición cuantitativa, debe considerarse una praxis fundamentada en la concepción del hombre. Lo anterior permite el reconocimiento de los individuos como sujetos activos y responsables, que hacen parte de procesos de desarrollo humano dentro de la esfera organizacional. Según Anzola (2018), han surgido nuevas formas de entender a las organizaciones, donde la gestión humana deja entrever capacidades de generación de nuevos saberes, diversas perspectivas de actuación, relaciones de poder, agentes de cambio, entre otros. Estos elementos amplían el enfoque organizacional, lo cual generalmente se encuentra demarcado por la mercantilización y la generación de rentabilidades.

El individuo, dentro del campo de la gestión humana, es un factor central en la generación de valor y diferenciación. Desde esta perspectiva, se considera pertinente adaptar modelos de gestión orientados a la heterogeneidad de los colaboradores, cuyo diferencial se encuentra en sus capacidades intrínsecas y su capacidad de contribuir al desempeño organizacional (Rivero \& Dabos, 2017).

Con base en los macro-procesos planteados por García et al. (2011), en la Figura 1 se explican las actividades que se desarrollan en gestión humana.

Figura 1: Los macro-procesos de Gestión Humana.

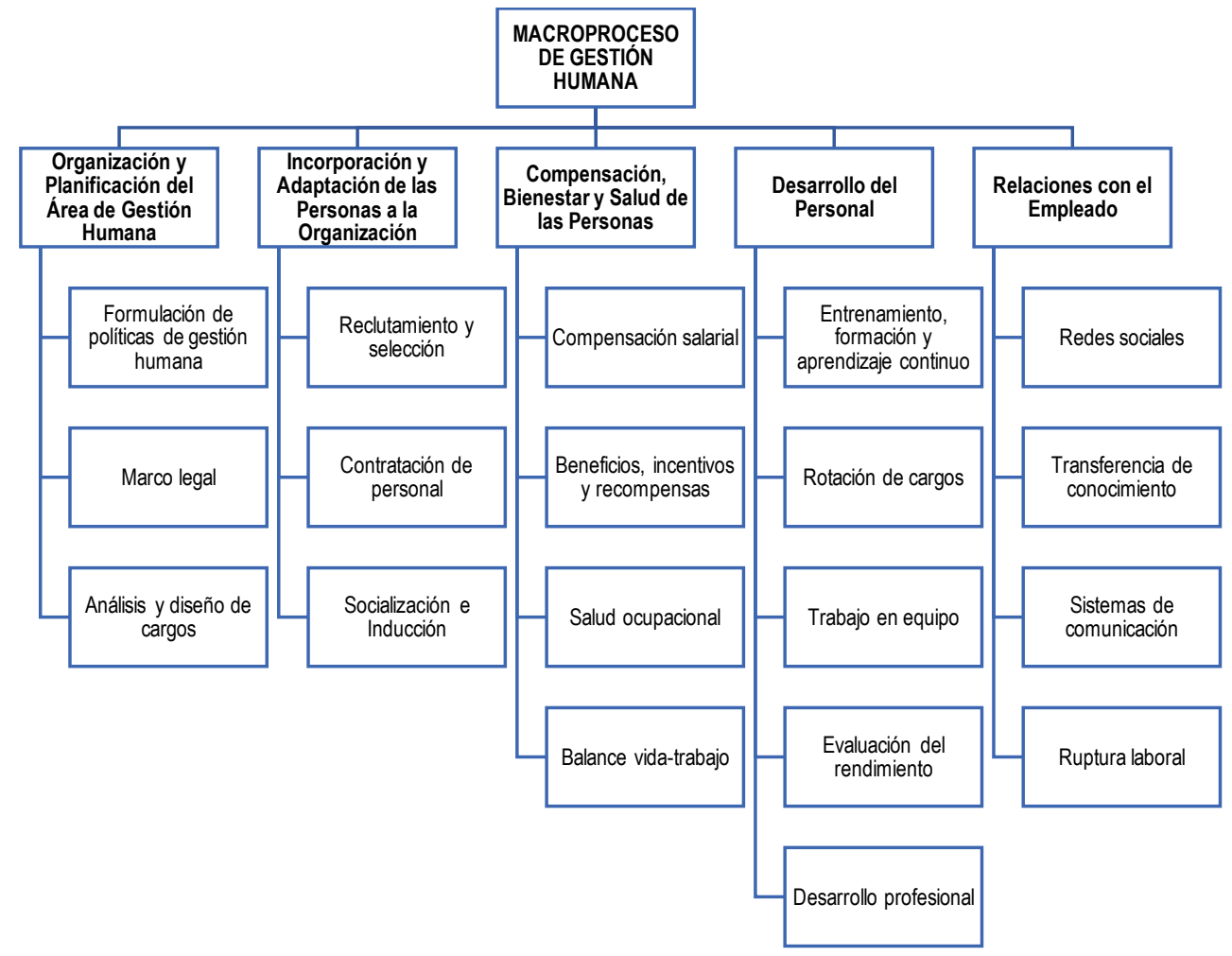

Fuente: Adaptado de García et al., 2011, p. 17. 
Las OIC cuentan con empleados, en los cuales, resaltan características muy destacadas. Algunas de estas características son niveles de formación educativa altos, o con recorridos largos dentro de áreas específicas del conocimiento, lo que les capacita para desenvolverse muy bien dentro de este tipo de organizaciones (Alvesson, 2001; 2004; Makani \& Marche, 2010; Starbuck, 1992; Swart \& Kinnie, 2003). De este modo, debe considerarse que, para las OIC, los colaboradores son los activos más importantes y deben estar alineados con los objetivos de creatividad e innovación requeridos para que este tipo de organizaciones se desarrollen tal y como esperan (Kemp, Moerman \& Prieto, 2001).

De acuerdo con Bruyne y Gerritse (2018), en los próximos 10 años se espera que los empleos existentes y las habilidades requeridas por las OIC sufran cambios sustanciales. Las habilidades personales futuras estarán asociadas con una mayor intensidad en el desarrollo de características como la creatividad, colaboración, innovación, emprendimiento y capacidades de adaptación. En cuanto a las habilidades técnicas, se espera que las personas posean sólidos conocimientos en tecnologías de la información y matemáticas. Se considera que las áreas de actuación más importantes y objeto de gran experticia girarán en torno a la seguridad de la información, robótica y cadenas de logística. Esto implica un desafío para la agenda de la gestión estratégica de los recursos humanos, la cual, tendrá que enfrentarse a retos como atraer y retener talento humano idóneo y alineado con las necesidades de las OIC, siempre que el mercado laboral sea capaz de responder a los requerimientos de especialización y complejidad del trabajo que suponen estas organizaciones.

El área de gestión del talento humano ha experimentado cambios en la esfera organizacional, con la introducción de nuevos modos de ejecución y en el desempeño de sus funciones. Si bien la perspectiva tradicional se ha centrado en proteger y apoyar el talento humano, en la actualidad se propende por entrenar a los colaboradores para conservar o incrementar el nivel de valor agregado que aporta con su trabajo. Las oportunidades de desarrollo del personal presentan una incidencia positiva en la generación de innovación, esto ha implicado una nueva concepción del trabajo donde se transita desde la definición de una función específica desempeñada por un colaborador, a destacar su contribución significativa a los equipos o proyectos a los cuales pertenece. En función de lo anterior, es importante resaltar que esta nueva concepción, a su vez, requiere condiciones de empleo mediadas por la flexibilidad y un sistema de clasificación de cargos fácilmente adaptable a los aspectos estructurales de la organización (Bruyne \& Gerritse, 2018).

Investigaciones como la de Camelo, García, Sousa y Valle (2011) muestran una relación positiva entre la gestión estratégica de los recursos humanos y la creación y transmisión de conocimiento en las OIC. Las actividades desarrolladas por la gestión humana no generan un impacto directo en el intercambio del conocimiento, pero si generan una disposición afectiva de compromiso con la organización. Es decir, aporta para que las personas compartan su experticia en distintas áreas, y de este modo, se genera conocimiento e innovación al interior de las organizaciones. No obstante, es necesario la vinculación de métodos alternativos, diferentes a las prácticas tradicionales de la gestión humana para conseguir que las personas se motiven a compartir y crear conocimiento e 
innovación, métodos que incentiven el desarrollo de la creatividad y la autonomía en los colaboradores.

El conocimiento colectivo se construye, a partir de la transformación del conocimiento individual, dicha transformación puede verse apoyada u obstaculizada por las prácticas de gestión humana (Mrinalini \& Nath, 2000). Por medio del intercambio de información entre las personas, se agrega valor a la misma. Los autores consideran que deben fomentarse actividades que permitan la participación colectiva, espacios para compartir sus ideas y experiencias, y trabajar en equipo. Se puede inferir, de este modo, que el talento humano es capaz de desarrollar, potenciar y enfocar su conocimiento y habilidades hacia el bien de la organización (Mrinalini \& Nath, 2000).

Según Juneja y Amar (2018), el capital humano se encuentra inmerso dentro del capital organizacional, y desde una perspectiva estrictamente económica, su nivel de especialización, procesos y prácticas son utilizados para transformar el conocimiento, generar mayor valor agregado en productos o servicios, y convertir la producción en futuros ingresos representativos para la OIC. Por otro lado, se considera al capital humano como generador de capital organizacional, a partir de habilidades personales, la interrelación entre el conocimiento que se posee fruto de la experiencia y el conocimiento estructurado en el que se encuentra inmersa la actividad humana y el modo en que las vivencias individuales aportan a la construcción del conocimiento colectivo.

Por consiguiente, Mabey y Zhao (2017) formulan una serie de prioridades distintivas para aquellas OIC, en las que el intercambio efectivo de conocimiento predominantemente tácito a partir de redes internacionales y globales son clave para alcanzar el éxito. Estas prioridades hacen referencia a la construcción de una estructura de confianza que a partir de diversos puentes de conocimiento, fortalecen la interdependencia y responsabilidades de los grupos; el enfoque en el proceso donde permanentemente se revisa el desempeño de los procedimientos; las recompensas generosas para quienes participan activamente en la construcción en redes internas y externas de conocimiento; el uso apropiado de plataformas TIC para la correcta gestión e interacción del conocimiento y el aumento de conciencia en la diversidad de las esferas culturales.

Para Jackson et al. (2006) para las OIC es importante centrarse en el conocimiento y el rendimiento, es aquí donde entra la gestión humana a jugar un papel muy importante porque debe asegurar que las competencias de los equipos de trabajo sean acordes a los objetivos de la empresa.

Se esperaría que la gestión del talento humano se encargara de algunos puntos muy específicos como: 1) Una rigurosa selección del personal que garantice la presencia de personas capaces de trabajar en entornos desafiantes y colaborativos; 2) Jornadas formativas y de desarrollo, tanto del individuo como de los equipos de trabajo, así como actividades de gestión del rendimiento que involucren al talento humano con los objetivos de la organización, y 3) Un sistema de retribución o remuneración con criterios definidos que funcione con base en el desempeño.

Comparando el trabajo del conocimiento con el trabajo tradicional, se pone en evidencia ciertas características que poseen los integrantes de las OIC, se les da una mirada desde 
la gestión de los recursos humanos desarrollada por Charles (1995). Estas características se muestran en la Tabla 1.

Tabla 1: Comparación Trabajo del Conocimiento y Trabajo Tradicional

\begin{tabular}{|c|c|c|}
\hline & Trabajo del Conocimiento & Trabajo Tradicional \\
\hline $\begin{array}{l}\text { Formación profesional del } \\
\text { empleado }\end{array}$ & $\begin{array}{l}\text { Externo a la organización, a } \\
\text { través de años de educación y } \\
\text { socialización }\end{array}$ & $\begin{array}{l}\text { Interno a la organización, a } \\
\text { través de entrenamiento, } \\
\text { desarrollo, desempeño, reglas } \\
\text { y planes de carreras } \\
\text { prescriptivos }\end{array}$ \\
\hline Lealtad del empleado & $\begin{array}{l}\text { A profesiones, redes y } \\
\text { compañeros }\end{array}$ & $\begin{array}{l}\text { A la organización y sus } \\
\text { sistemas de carrera }\end{array}$ \\
\hline $\begin{array}{l}\text { Habilidad / Conjunto de } \\
\text { conocimientos }\end{array}$ & $\begin{array}{l}\text { Especializado y profundo, pero } \\
\text { siempre con focos periféricos } \\
\text { difusos }\end{array}$ & Estrecho y a menudo funcional \\
\hline Locus de Trabajo & En grupos y proyectos & Individuales \\
\hline Enfoque de Trabajo & Clientes, problemas, temas & Tareas, objetivos, desempeño \\
\hline $\begin{array}{l}\text { Obsolescencia de las } \\
\text { Habilidades }\end{array}$ & Rápida & Gradual \\
\hline $\begin{array}{l}\text { Actividad / Ciclos de } \\
\text { Retroalimentación }\end{array}$ & $\begin{array}{l}\text { Larga desde una perspectiva } \\
\text { empresarial }\end{array}$ & $\begin{array}{l}\text { Primaria y de una naturaleza } \\
\text { inmediata }\end{array}$ \\
\hline Medidas de Desempeño & $\begin{array}{l}\text { Proceso de eficacia, } \\
\text { potencialmente grande, pero } \\
\text { siempre errático }\end{array}$ & $\begin{array}{l}\text { Presentaciones de las tareas, } \\
\text { pequeño (según lo previsto), } \\
\text { pero regular y confiable }\end{array}$ \\
\hline $\begin{array}{l}\text { Impacto en el Éxito de la } \\
\text { Empresa }\end{array}$ & $\begin{array}{l}\text { Pocas contribuciones de } \\
\text { mayor importancia estratégica } \\
\text { y a largo plazo }\end{array}$ & $\begin{array}{l}\text { Muchas pequeñas } \\
\text { contribuciones que apoyan el } \\
\text { plan maestro }\end{array}$ \\
\hline
\end{tabular}

Fuente: Adaptado de Charles, 1995, p. 9-23.

Las características que se asocian a los trabajadores de las OIC, según Greenwood (2009), son Inteligente, autocontrolado y autoevaluado; esto se ha determinado bajo algunas premisas que conciben al colaborador como el depositario del conocimiento, por sus valores orientados al constante aprendizaje, y el fomento y desarrollo continuo de dicho conocimiento. Algunos autores han realizado revisiones literarias para definir, de una manera más amplia, las características de los trabajadores de estas organizaciones intensivas en conocimiento.

Makani y Marche (2010), por ejemplo, explican que tras su estudio se hacen evidentes las altas capacidades de estos colaboradores en materia profesional; capacidad superior para gestionar situaciones y resolver conflictos que se presenten en todos los ambientes de trabajo; tienen muchas habilidades blandas y duras, así como creatividad e innovación; apropiación de temas de forma conceptual y cognitiva; son educados, calificados y cualificación para desempeñarse de forma eficiente dentro de la organización, etc. (Alvesson, 2001; 2004; Ichijo \& Nonaka, 2006; Starbuck, 1992; Sveiby \& Lloyd, 1987).

De estos trabajadores se perciben todos estos aspectos, no sólo por la adquisición de ellos de forma individual, sino que, además, muchas de ellas han sido formadas y desarrolladas por el contexto en el que se desempeñan. (Carmona et al., 2005). En la Figura 2, se presenta dicho contexto: 
Figura 2: Contexto Social del Trabajador del Conocimiento

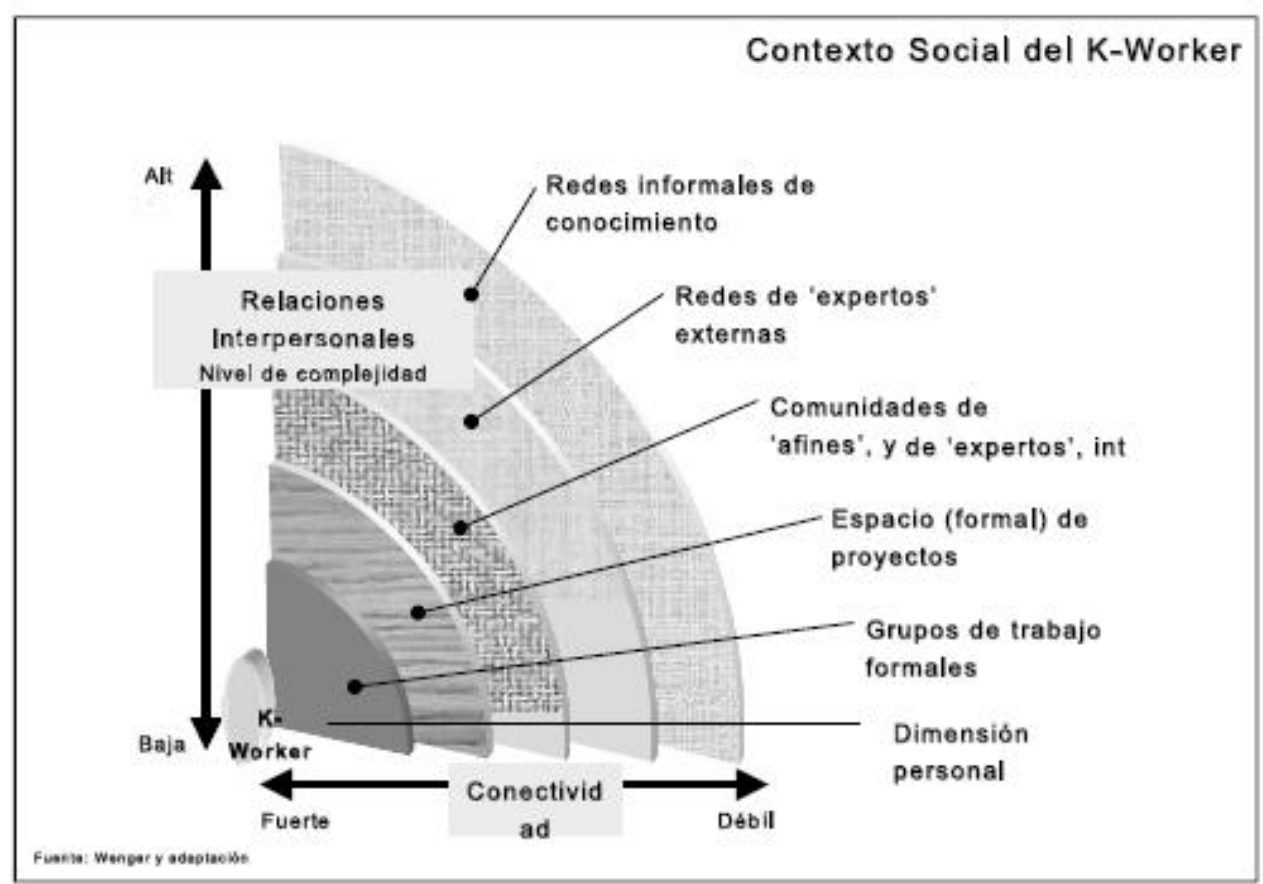

Fuente: Adaptado de Carmona, Nieto y Rubio, 2005, p.233-252.

Drucker (1993), acuña un término para denominar a la sociedad actual, él la llama "la sociedad post-capitalista o sociedad del conocimiento"; plantea que, para esta sociedad, el único recurso económico que posee en sí un significado es la información, la cual, es generadora de conocimiento y es aquí donde está consignado el ejercicio principal de las OIC.

La capacidad del talento humano y el aprovechamiento de los recursos físicos, hacen posible el acceso y el correcto procesamiento y utilización de la información que actúa como un impulso generador de conocimiento en las organizaciones. El sistema de conocimiento se ve enriquecido por aquellos individuos que ponen al servicio de las metas y objetivos organizacionales su conocimiento, de este modo, se les puede llamar parte de los recursos humanos (Mrinalini \& Nath, 2000).

De acuerdo con Gope, Elia y Passiante (2018) la gestión estratégica del talento humano de las OIC y sus diversas actividades, garantizan la sostenibilidad conforme al desarrollo de habilidades específicas de capital humano, y la creación de conocimiento valioso que puede ser explotado conforme a los lineamientos de la estrategia organizacional. En la medida que la gestión del talento humano y la gestión del conocimiento estén alineadas, se incrementa el aprendizaje organizativo que configura procesos de innovación y sostenibilidad, en el tiempo, frente a su competencia. En ese sentido, el conocimiento y las competencias deben ser abordados a plenitud desde las políticas y planes de acción de la gestión estratégica del talento humano, con el fin de garantizar que la fuerza laboral se encuentre permanentemente actualizada y su rol sea conducente a los objetivos organizacionales. 


\section{METODOLOGÍA}

Tras la realización de un proyecto de investigación llevado a cabo en 4 fases, surge como resultado el presente artículo. En la primera fase, se realiza una búsqueda bibliográfica sobre las consideraciones conceptuales acerca de las OIC y los modelos de gestión humana, especialmente los macroprocesos. Las consultas fueron realizadas en bases de datos electrónicas mediante búsquedas con palabras claves que permitieron encontrar artículos relevantes que fueron clasificados por enfoques y por fecha de publicación. Desde su lectura se formuló el estado del arte y el marco teórico presentado. En la segunda fase, utilizando la metodología de estudio de caso propuesta por Yin (2003), de manera individual se aborda la caracterización de cada CIE, como un caso, este abordaje es realizado combinando revisión documental con entrevistas estructuradas a los responsables de los $\mathrm{CIE}$, los resultados fueron sistematizados y organizados. En la tercera fase, mediante comparativos, se establecen semejanzas y diferencias entre los procesos de gestión humana con implementados cada CIE. En la cuarta fase, se construye caracterización de algunos macroprocesos de gestión humana para organizaciones intensivas de conocimiento, partiendo de los comparativos realizados que son contrastados con los hallazgos encontrados en la revisión documental y el estado del arte. La siguiente figura es una representación gráfica de la metodología de estudio de caso utilizada en la caracterización de la estructura organizacional de cada CIE.

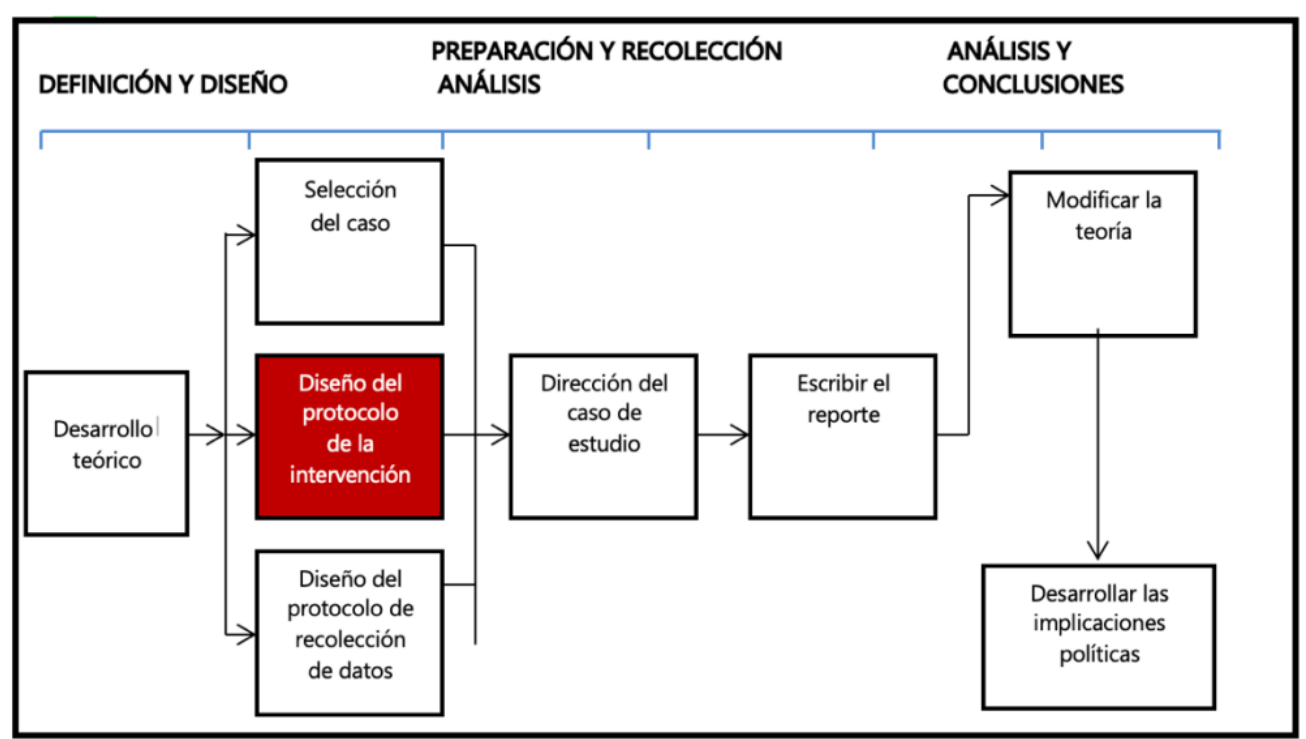

Figura 1. Estructura de Estudio de Caso. Tomado de González, García y Murillo (2016, p. 103).

La metodología propuesta ha permitido la validez de los resultados, tanto por el método de los estudios de casos, como por los instrumentos de recolección y análisis de datos. El resultado, aunque tiene las particularidades y las limitaciones de los casos seleccionados, en el país, son las organizaciones intensivas en conocimiento, de mayor relevancia por la producción de nuevo conocimiento que durante su existencia los ha posicionado entre la comunidad científica, razón por la cual, se convierte en hallazgos representativos que pueden ser replicados en contextos similares, con una mirada desde el ámbito de las organizaciones, como en otros países o regiones. 


\section{RESULTADOS}

A continuación, se mencionarán 5 Centros de investigación todos inscritos a Colciencias y se mencionará la manera cómo funcionan sus procesos de gestión humana llevados a cabo durante su periodo de existencia. Dichos Centros de investigación son: GEBIX (Genómica y Bioinformática de Ambientes Extremos); CENM (Centro de Excelencia en Nuevos Materiales); CCITB (Centro Colombiano de Investigación en Tuberculosis); CENIVAM (Agroindustria de Especies Vegetales Aromáticas y Medicinales Tropicales); CIEBREG (Biodiversidad Y Recursos Genéticos).

Los Centros de investigación en Colombia se conforman de acuerdo a los términos de referencia de la convocatoria del Centro de Excelencia por Colciencias, bajo Unión Temporal, es decir, un acuerdo entre 2 o más grupos de investigación o instituciones educativas, como, por ejemplo, Universidades. Estos actores deben responder solidariamente ante el cumplimiento de lo propuesto en el proyecto y para obtener el NIT, deben cumplir algunas obligaciones tributarias que requieren tramitar su registro único tributario. Un ejemplo de lo anterior es el Centro GEBIX conformado por siete (7) miembros; Corporación Corpogen, Parquesoft, Universidad Nacional, Universidad del Valle, Universidad del Cauca, Universidad de Caldas y Universidad Javeriana. El CIEBREG, por otro lado, cuenta con una agrupación de 5 instituciones que entre ellas conforman 7 grupos de investigación.

Estos Centros de investigación cuentan con periodos de existencia fijos, es decir, terminan pasados un número específico de años, dependiendo de la convocatoria duran menos o más años, en el caso de GEBIX este periodo es de únicamente dos (2) años, en el caso de CENM dura cinco (5) años, en el caso de CCITB duran cinco (5) años, en el caso de CIEBREG cinco (5) años. Esto impide por ejemplo pensar en suscribir contratos laborales a término indefinido. Este tipo de situación ha llevado a los Centros de investigación a ser claros al comunicar los tiempos de duración del contrato y no hacer falsas promesas sobre planes de carrera. Un ejemplo de lo anterior es GEBIX con los siguientes lineamientos:

a. No crear falsas expectativas dado el carácter temporal de GEBIX, es decir se dificulta hablar de planes de carrera, pues esto significa permanencia a largo plazo.

b. En la medida de lo posible, la celebración de un nuevo contrato cuando un investigador ha cumplido a cabalidad sus actividades, sí: el requerimiento de nuevos servicios implica una continuidad con servicios desempeñados con anterioridad; 2. Para llevar a cabo servicios en el futuro, es necesaria la continuidad; 3. El enfoque técnico o científico necesita tener una línea continua; 4. Cuando, es preferible continuar con el contratista inicial dada la experiencia adquirida y la responsabilidad profesional, antes que adelantar un nuevo proceso competitivo, por supuesto, bajo la condición de que el trabajo desempeñado anteriormente haya cumplido con las expectativas esperadas.

c. Asegurar la permanencia de los investigadores principales durante el tiempo de ejecución del proyecto como compromiso de cada una de las instituciones de GEBIX. 
Como toda empresa constituida, los Centros de investigación deberían, desde un vistazo administrativo, contar con un organigrama que permita aclarar posiciones jerárquicas, quién esta abajo, quién está arriba y en qué departamento está ubicado, para así mismo desarrollar un diseño del cargo que responda y sea pertinente a las necesidades del Centro. Pero en este caso, se observa, precisamente como debilidad, que los Centros de Investigación suelen no contar con dichas estructuras, la razón principal, aunque no es regla para todos, es porque desde los grupos que conforman al Centro estos organigramas ya existen, así como el proceso de selección de cada grupo. Es así como en el GEBIX, no hay organigrama y con independencia en los términos de referencia para las contrataciones por cada grupo que le conforma, donde se especifican la justificación, el objeto, las características técnicas el perfil profesional requerido y las obligaciones especificas del contratista, así como independencia en el proceso de selección que en términos generales para todo el grupo, se basa en criterios de experiencia, entrevistas y recomendaciones y está a cargo el investigador principal; CENM, donde la responsabilidad del proceso de selección recae únicamente en los grupos de investigación vinculados al Centro, y el organigrama que existe fue plasmado desde las propuestas de los grupos únicamente al inicio de la constitución por lo cual los nuevos cargos que van surgiendo como necesidad, simplemente no se pensaron. CCITB por ejemplo, no define sus administradores, sino que este proceso de selección es dirigido por la Corporación para Investigaciones Biológicas que a su vez administra al Centro y por otro lado los cargos de investigación, personal de laboratorio y de estudiantes en formación son definidos por cada uno de los grupos de investigación.

Acerca de los tipos de vinculación, los Centros de Investigación suelen regirse, debido a su existencia temporal, a un tipo de vinculación por prestación de servicios que suelen ser los mismos investigadores, estos a su vez son supervisados por los investigadores principales que vienen desde los grupos de investigación que conforman el Centro, es decir que estos últimos no son contratados por el Centro, sino que vienen desde una contratación previa, así como los cargos administrativos. En este sentido para el GEBIX se contaba con cargos de contratistas que son los biólogos, abogados, ingenieros de sistemas y demás profesionales que se contrataron por prestación de servicios y los demás cargos como el investigador principal y los administrativos, como la coordinadora administrativa y la directora del Centro, son diferentes contratos, algunos previos a la creación del Centro. Sin embargo, existen casos en los que se da otro carácter de vinculación diferente a la prestación de servicios, en el CENM que contaba con tipos de vinculación como contrapartida y monitoria, lo cual genera una fortaleza para el Centro en el manejo de las contrataciones por proyecto. CCITB, por otro lado, tiene como modalidades de vinculación: definido, indefinido y estudiantes en formación.

Sobre la capacitación es normal no encontrarla en los Centros como plan formal, ya que como se ha mencionado, el proceso de selección está a cargo de los grupos o investigadores principales que componen al Centro. Es así como en el CCITB no se contaba con un plan de capacitación formal pero en lo que respecta a sus unidades temáticas cuenta por cada una de ellas con actividades de entrenamiento en técnicas de laboratorio y manejo de herramientas (software y programas de análisis) entre otros talleres realizados en encuentros de investigadores a nivel internacional, otro ejemplo de un plan de capacitación no definido, pero que aun así existían en el Centro es el de CENIVAM, donde los investigadores reciben capacitación en técnicas de análisis y en el 
manejo de equipos. Así mismo el Centro CIEBREG, contaba con un programa de capacitación no formal por grupos de personal que recogía tanto a investigadores como al personal administrativo, concluyendo en un total de 46 eventos de capacitación y formación.

En el caso de estudio, se establece que desde la gestión humana se buscaba incentivar al personal a realizar un mejor trabajo siempre que sea posible, el objetivo puede ser mejorar el rendimiento o crear afiliación hacia la empresa, otras veces puede ser el simple desarrollo como profesional del colaborador, en fin, suelen existir varias razones por las que se incentiva al personal. Para los Centros de Investigación, la razón principal es el crecimiento profesional y el apoyo de este nuevo conocimiento a los proyectos, dejando a un lado la afiliación pues se conoce de antemano el carácter temporal del Centro. Ejemplos de los incentivos que otorgan los diferentes Centros son: GEIBX, donde se financió la inscripción de la gran mayoría de investigadores en el Curso de Metagenómica y Bioinformática; CENM y CIEBREG, que brindan apoyo a través de proyectos para estudios de maestría y doctorado, cada uno con sus respectivas pasantías, este tipo de proyectos son realizados mediante convocatoria abierta. Por otro lado, existen Centros que no brindan incentivos a los investigadores por prestación de servicios, solo reciben incentivos los investigadores principales o investigadores que tienen vínculos laborales con las universidades en relación de docencia o investigación tal y como es el caso de CCITB. Desde otra perspectiva también se llega a incentivar a los cargos operativos o de logística como es el caso de CENIVAM donde se incentiva a los jardineros y a los de oficios varios a mejorar su nivel académico, ofreciéndoles la oportunidad de ampliar sus estudios.

Lo concerniente con la puesta en marcha de las actividades muestra la flexibilidad y autonomía de los investigadores en los Centros, de los cuales hay quienes no tienen sedes principales y por ende trabajan desde las diferentes sedes de los grupos a los que pertenecen. De esta forma en el GEBIX no se contaba con sede principal y los investigadores y algunos colaboradores no cuentan con horarios fijos y realizan sus actividades de forma descentralizada. Este tipo de contratación atrae personal calificado interesado en participar en los proyectos, pero, por otro lado, no es fácil retenerlos por la incertidumbre frente a la permanencia en futuros proyectos. Otro ejemplo es CENIVAM donde no se contaba con sede principal y, por ejemplo, las actividades de higiene y seguridad industrial son realizadas donde está ubicado cada grupo de investigación. Desde este aspecto de actividades se puede mencionar también la evaluación o medición de desempeño de las mismas, ya que como se ha destacado, la flexibilidad existente se basa principalmente más que en horarios en resultados, es decir, en la entrega de artículos o avances de los mismos, en Centros como GEBIX la evaluación de desempeño la hace el investigador principal a los investigadores de menor rango, en el Centro de CCITB no se mide igual a los investigadores ni se les exige productos entregables, sin embargo a los estudiantes, si se les exige una entrega ya sea de artículos, presentaciones y/o posters en eventos nacionales e internacionales, como soporte de la financiación que reciben, y en general el desempeño se mide desde la cantidad de compromisos cumplidos, que son pactados por cada investigador principal al inicio de cada año.

Finalmente, en el estudio se identifica que, en la estructura salarial de los Centros de Investigación de Excelencia, no se percibía por el trabajo desempeñado un salario fijo, 
sino el pago de honorarios posteriores al desarrollo de las actividades delegadas, las cuales, eran aceptadas o no, por un investigador principal quien decide si el trabajo pasa con correcciones o simplemente no pasa. Ejemplos de lo anterior eran el GEBIX y el CCITB entre otros, que cuentan con honorarios que eran establecidos teniendo en cuenta las tablas de Colciencias además de la formación académica y la experiencia requerida para el cargo.

Con lo anteriores hallazgos en el estudio de los CIE en Colombia, y realizando una contrastación con la teoría desarrollada y los estudios previos encontrados, se presentan en la Tabla 2, una caracterización general de cada macroproceso de Gestión Humana para las Organizaciones Intensivas de Conocimiento.

Tabla 2 Gestión humana en las OIC

\begin{tabular}{|c|c|c|}
\hline Proceso & Característica & Autores \\
\hline $\begin{array}{l}\text { Reclutamiento } \\
\text { y selección }\end{array}$ & $\begin{array}{l}\text { El reclutamiento describe los métodos y procedimientos } \\
\text { por los cuales se atrae personal especializado a una OIC. } \\
\text { En ese sentido, el proceso de selección establece los } \\
\text { criterios personales, de profesionalización y experiencia, } \\
\text { para escoger colaboradores integrales con actitud y } \\
\text { potencial para aprender. En el caso de las } \\
\text { organizaciones de conocimiento y específicamente los } \\
\text { centros de excelencia estudiados que son uniones } \\
\text { temporales de grupos de investigación dicho macro- } \\
\text { proceso se centra en el estudio de los proyectos a } \\
\text { desarrollar y a partir de ellos se identificaran el personal } \\
\text { que se requiere para su ejecución, el tiempo y tipo de } \\
\text { contratación que se debe manejar, estos procesos los } \\
\text { desarrollara el director del centro en conjunto con un } \\
\text { asistente administrativo y definirán el número de } \\
\text { personal requerido y el perfil de las personas acordes a } \\
\text { las funciones que se requieran dentro de cada proyecto, } \\
\text { teniendo en cuenta los productos a entregar }\end{array}$ & $\begin{array}{l}\text { Medina y Medina } \\
\text { (2017), lqbal et al. } \\
\text { (2015), Kaiser, } \\
\text { Kozica, Swart y } \\
\text { Werr (2015) }\end{array}$ \\
\hline Adaptación & $\begin{array}{l}\text { En el caso de las organizaciones de conocimiento y } \\
\text { específicamente los centros de excelencia estudiados. El } \\
\text { proceso de reclutamiento y selección está caracterizado } \\
\text { por buscar personas que aporten al desarrollo de nuevo } \\
\text { conocimiento y a los procesos de aprendizaje y } \\
\text { formación de las personas que participaran en los } \\
\text { proyectos a desarrollar. Por lo tanto, el proceso de } \\
\text { reclutamiento y selección en su perfil primara el } \\
\text { conocimiento que tienen los candidatos y las habilidades } \\
\text { blandas que posean para poder trabajar en equipo, } \\
\text { desarrollar procesos con liderazgo y buenas relaciones } \\
\text { interpersonales. Adicionalmente un factor importante a } \\
\text { medir será la experiencia que se tiene en el desarrollo } \\
\text { de proyectos y en la generación de conocimientos y el } \\
\text { nivel de formación que se tiene. El proceso de selección } \\
\text { deberá incluir pruebas psicotécnicas y pruebas de } \\
\text { conocimiento, más entrevista con el coordinador del } \\
\text { proyecto y el director del centro. El proceso de } \\
\text { inducción permite una mejor adaptación y generación } \\
\text { de sentido de pertenencia de las personas, como los }\end{array}$ & $\begin{array}{l}\text { Corcoran y Duane } \\
\text { (2018) }\end{array}$ \\
\hline
\end{tabular}


tiempos en la organización son definidos, es importante que se realice un proceso donde se le explique y se le dé a conocer la organización, los cargos, las personas y el proyecto, más los productos esperados y de esta manera generar el conocimiento de las actividades a desarrollar. El proceso de contratación es por tiempo definido y acorde a la reglamentación legal vigente.

\begin{tabular}{|c|c|c|}
\hline Capacitación & $\begin{array}{l}\text { Los procesos de capacitación proveen herramientas } \\
\text { para el intercambio y creación de nuevo conocimiento. } \\
\text { Estos deben estar ajustados a la realidad organizacional } \\
\text { y a las necesidades de aprendizaje de los colaboradores. }\end{array}$ & $\begin{array}{l}\text { AlShamsi y Ajmal } \\
\text { (2018), Khanbabaei } \\
\text { et al., (2019), Ardito } \\
\text { y Messeni (2017) }\end{array}$ \\
\hline $\begin{array}{l}\text { Bienestar } \\
\text { laboral }\end{array}$ & $\begin{array}{l}\text { En el caso de las organizaciones de conocimiento y } \\
\text { específicamente los centros de excelencia estudiados. La } \\
\text { estructura salarial dependerá de los recursos disponibles } \\
\text { en cada uno de los proyectos y se hará jerarquizada por } \\
\text { nivel de formación y conocimiento o de acuerdo a los } \\
\text { productos a entregar, por lo que el sistema de incentivos } \\
\text { y beneficios es mínimo, se debe trabajar más desde } \\
\text { procesos de motivación no económica como por } \\
\text { ejemplo el tiempo laboral flexible, procesos de } \\
\text { aprendizaje y formación, el obtener recursos de acuerdo } \\
\text { a los productos o resultado esperado, este conjunto de } \\
\text { factores movilizan los cambios hacia una percepción de } \\
\text { estabilidad y buenas condiciones en el trabajo. Con } \\
\text { relación a los procesos de salud ocupacional } \\
\text { independiente del tipo de contratación que se tenga hoy } \\
\text { en día se debe cumplir con todo lo reglamentado. }\end{array}$ & $\begin{array}{l}\text { Kamaruddin et al., } \\
\text { (2018), Mabey y } \\
\text { Zhaor (2017), } \\
\text { Arunprasad, (2017), } \\
\text { Kaiser et al., (2015) }\end{array}$ \\
\hline $\begin{array}{l}\text { Desarrollo y } \\
\text { formación }\end{array}$ & $\begin{array}{l}\text { Los procesos de formación y capacitación son esenciales } \\
\text { en el desarrollo de los proyectos. Es necesario, además, } \\
\text { realizar un diagnóstico de las capacidades que se tienen } \\
\text { y las ausentes, para el logro de los objetivos. Estos } \\
\text { procesos están más orientados a competencias } \\
\text { específicas y no a las competencias blandas, es muy } \\
\text { probable que haya desarrollo en las personas a nivel } \\
\text { personal y profesional pero no existe un plan carrera, } \\
\text { debido a que las contrataciones son por tiempos } \\
\text { definidos, aunque puede haber continuidad en la } \\
\text { contratación para un nuevo proyecto de acuerdo a los } \\
\text { resultados obtenidos, la evaluación del desempeño se } \\
\text { realiza de acuerdo al producto terminado requerido en } \\
\text { los contratos a término fijo, o prestación de servicio y el } \\
\text { cumplimiento de metas propuestas. El seguimiento se } \\
\text { realiza a través de una base de datos donde se almacena } \\
\text { la historia de las personas que se han contratado y sus } \\
\text { capacidades y habilidades. }\end{array}$ & $\begin{array}{l}\text { Bruyne y Gerritse, } \\
\text { (2018), Moon et al., } \\
\text { (2016) }\end{array}$ \\
\hline $\begin{array}{l}\text { Relaciones } \\
\text { laborales }\end{array}$ & $\begin{array}{l}\text { En el caso de las organizaciones de conocimiento y } \\
\text { específicamente los centros de excelencia estudiados, } \\
\text { no existen proceso de negociación colectiva o sindicales } \\
\text { de manera formal, el proceso de cesación o ruptura se } \\
\text { da generalmente por finalización del contrato o por el } \\
\text { no cumplimiento de los productos requeridos. }\end{array}$ & $\begin{array}{l}\text { Parmer y Dillard } \\
\text { (2018), Stenius et } \\
\text { al., (2017), Dal Mas } \\
\text { et al. (2018) Millar, } \\
\text { Chen y Waller } \\
\text { (2017) }\end{array}$ \\
\hline
\end{tabular}

Fuente: Elaboración propia. 
De este modo, la creación, intercambio y aporte de conocimiento de los trabajadores y el desarrollo de sus habilidades, la satisfacción que sientan por su trabajo y su retención deben estar soportados por la gestión estratégica del talento humano en las OIC, que a su vez están agrupados según García et al. (2011) en los cinco macroprocesos de Gestión Humana.

En este contexto, retomando Gope et al. (2018), cuando la gestión del talento es Ilevada a cabo mediante procesos de reclutamiento, actividades de desarrollo de personal, un efectivo sistema de retención de empleados, implementación de modelos de liderazgo que fortalezcan la relación entre directivos - colaboradores, gestión del rendimiento y un sistema de recompensas acorde con las capacidades organizacionales, se logra un impacto positivo de las actividades de gestión humana sobre la obtención de rendimientos tanto funcionales como financieros en una OIC.

Por esta razón, el fortalecimiento de las relaciones con los empleados también es posible en tanto la organización cuida sus necesidades y su bienestar. En ese sentido, la satisfacción de las necesidades psicológicas de los colaboradores debe ser tenidas en cuenta por los encargados de gestión humana en la empresa, y deben percibirse como un medio, por el cual, se puede fomentar el intercambio de conocimiento, a partir de prácticas de gestión basadas en la cualificación del personal. De esta manera, un colaborador al sentirse competente por contribuir significativamente a la organización, autónomo para tomar decisiones que respalden los objetivos de su cargo y con un alto grado de pertenencia para sentirse socialmente conectado con el trabajo, es más propenso a adoptar actitudes y conductas que busquen el beneficio colectivo (Stenius, Haukkala, Hankonen, \& Ravaja, 2017)

Para generar oportunidades que contribuyan a la construcción del conocimiento, las organizaciones deben, según lo propuesto por Pérez et al. (2009), reducir las barreras que generan distanciamiento entre los colaboradores, tanto física como psicológicamente. Resulta vital, en las OIC permitir la comunicación en todas las direcciones y entre los distintos niveles jerárquicos de la compañía, fomentando el intercambio de ideas a través de sistemas de comunicación efectivos, que además de facilitar la comunicación entre las partes, mantenga al colaborador al tanto de los objetivos organizacionales tanto generales como las estrategias del presente. Esta creación de nuevo conocimiento se alcanza por los caminos de la construcción de un buen clima organizacional, que, a su vez, impacta en la creación de redes de trabajo y fomenta el trabajo cooperativo, así como la adopción de comportamientos, lenguajes, misiones y visiones comunes, que entran a mediar los aportes individuales y el intercambio en las relaciones. Lo que en el momento del estudio no se identificó de manera explícita en los procesos implementados en los CIE.

En estas organizaciones se plantea un reto para la gerencia, se ubica en la forma de controlar la influencia que tienen los colaboradores en la dirección y viceversa, puesto que en las OIC se evidencia mayor libertad y responsabilidades otorgadas a los trabajadores (Kemp et al, 2001). Nonaka y Takeuchi (1995) sugieren crear una visión colectiva, es decir, que las actividades realizadas, satisfagan las necesidades organizacionales. 
La caracterización de los macroprocesos de gestión humana en los CIE es el inicio de una propuesta que se podrá implementar en algunas organizaciones intensivas de conocimiento, para que, como lo han planteado los diferentes autores, los colaboradores de estas organizaciones cumplan el rol requerido, pero al mismo tiempo para que los líderes de las OIC cuenten con las características de cada macroproceso para la toma de decisiones y la definición de estrategias de gestión humana eficaces.

\section{CONCLUSIONES}

La gestión estratégica de los recursos humanos y las actividades que desarrolla, están enfocadas en el trabajo con las organizaciones tradicionales, como las empresas, compañías manufactureras y de servicios, sin embargo, no dejan de ser generadoras de ventajas competitivas, factor importante para cualquier tipo de organización, incluso las OIC, ya que le permiten posicionarse frente a entes académicos nacionales e internacionales.

La dirección de la OIC que desee desarrollar ventajas competitivas a corto plazo y rendimientos de largo plazo debe apoyarse en los procesos de gestión humana. Como primera instancia, debe considerar el factor humano con el que cuenta, atendiendo las necesidades que identifique y aquellas que pueda satisfacer en el beneficio de la relación empresa-colaborador, que como se mencionó anteriormente termina redituando en el beneficio de la organización en general.

Para estos autores y según lo encontrado en los CIE, la gestión humana en sus procesos de selección debe estar atenta a aquellos individuos que estén dispuestos a crear nuevo conocimiento y que disfruten del constante aprendizaje, características requeridas en el perfil de los colaboradores de las OIC. Este proceso riguroso se da, sobre todo, cuando la organización ha hecho un trabajo de identificación de las carencias en conocimientos que tiene, por tanto, espera atraer el personal que contribuya a cerrar la brecha de conocimiento, formación y experiencia que aún se manifieste ausente.

Hace falta reconocer las prácticas de la gestión humana que crean y transfieren conocimiento en los trabajadores, y que son más profundas que la relación limitada a la formación y compensación, pues consiste en factores invisibles de las personas, que, por lo general, buscan desarrollarse exitosamente en el trabajo y en su vida personal. Dichas prácticas retienen ese talento humano de mayor calidad en el mundo laboral y las mantienen motivadas y comprometidas con la organización.

Para el caso de las Organizaciones Intensivas en Conocimiento, no es muy común que se den asociaciones sindicales, por tanto, el macroproceso de relaciones con el empleado antes que incluir el factor de relación con los sindicatos, debería fortalecer la relación empresa-colaborador, entre colaboradores y resolución de conflictos. Así, la creación y transferencia de conocimiento, que se mencionó anteriormente, es un activo vital en este tipo de organizaciones, se obtendrá por la vía de la experiencia individual compartida y potenciada por la interacción entre las partes involucradas de la OIC.

Los resultados presentados en este artículo se convierten en un aporte al conocimiento desde la reflexión de los macroprocesos de gestión humana para organizaciones 
intensivas de conocimiento, pero también para la discusión existente en la actualidad del rol de los trabajadores en la evolución de las organizaciones, y los requerimientos que este rol, nuevo de alguna forma en los estudios sobre la gestión del talento humano, impone sobre la dirección estratégica de las organizaciones.

\section{REFERENCIAS}

AlShamsi, O., \& Ajmal, M. (2018). Critical factors for knowledge sharing in technology-intensive organizations: evidence from UAE service sector. Journal of Knowledge Management, 22(2), 384-412. https://doi.org/10.1108/JKM-05-2017-0181

Alvesson, M. (2001). Knowledge Work: ambiguity, image and identity. Human Relations, 7(54), 863 886.

Alvesson, M. (2004). Knowledge-work and Knowledge Intensive Firm. Oxford, UK: Oxford University Press.

Alvesson, M. (2000). Social Identity and the Problem of Loyalty in Knowledge-Intensive Companies. Journal of Management Studies, 37(8), 0022-2380.

Anzola, O. (2018). La gestión de recursos humanos y la construcción de subjetividades en las nuevas condiciones de la economía. Universitas Psychologica, 17(1), 1-14. https://doi.org/10.11144/javeriana.upsy17-1.grhc

Ardito, L., \& Messeni, A. (2017). Breadth of external knowledge sourcing and product innovation: The moderating role of strategic human resource practices. European Management Journal, 35(2), 261-272. https://doi.org/10.1016/j.emj.2017.01.005

Arunprasad, P. (2017). Inevitable knowledge strategy: a paradigm shift in strategic HRM practices to augment firm's performance. Employee Relations.

Boxall, P. F., \& Purcell, J. (2003). Strategy and human resource management. Houndmills, Basingstoke, Hampshire: Palgrave Macmillan.

Bruyne, E., \& Gerritse, D. (2018). Exploring the future workplace: results of the futures forum study. Journal of Corporate Real Estate, 20(3), 196-213. https://doi.org/http://dx.doi.org/10.1108/MRR$\underline{09-2015-0216}$

Camelo, C., García, J. \&Sousa, E. (2011). The influence of human resource management of knowledge sharing and innovation in Spain: the mediating role of affective commitment. International Journal Of Human Resource Management, 22(7), 1442-1463.

Calvo, N. (2007). Análisis de las prácticas de reclutamiento y selección como políticas de gestión del conocimiento: un enfoque desde la teoría de los recursos y capacidades. En Congreso Anual de AEDEM, Vol. 2, pp.21. Palma de Mallorca.

Carmona, E.; Nieto, W.; y Rubio, E. (2005). Entorno web del trabajador del conocimiento adecuado a las necesidades de organizaciones intensivas en conocimiento: caso centros I+D+I. Revista Iberoamericana de educación a Distancia, 8 (2), 233-252.

Charles, J. (1995). Human resource management in the knowledge age: current practice and perspectives on the future. Employee Relations, 17(1), $9-23$.

Corcoran, N., \& Duane, A. (2018). Using Social Media to Enable Staff Knowledge Sharing in Higher Education Institutions. Australasian Journal of Information Systems, 22, 1-26. https://doi.org/10.3127/ajis.v22i0.1647

DeCenzo, D. A., y Robbins, S. P. (2001). Administración de recursos humanos. México, D.F: Limusa. 
Dessler, G. (1991). Administración de personal. Mexico: Englewood Cliffs, N.J.

Drucker, P. (2002). La Gerencia en la Sociedad Futura. Bogotá: Editorial Norma.

Drucker, P. (2003). La Gestión del Conocimiento. Madrid: Ediciones Deusto.

García, M. (2008). La Gestión Humana en las organizaciones: Una perspectiva teórica. En: García, M., Sánchez, K., \& Zapata, A. (2008). Perspectivas Teóricas para el Estudio de la Gestión Humana (pp. 13-65). Santiago de Cali: Editorial Universidad de Valle.

García, M.; Murillo, G.; y González, C. (2011). Macroprocesos: Un nuevo enfoque al estudio de la gestión humana. Santiago de Cali: Editorial Universidad del Valle.

Gope, S., Elia, G., \& Passiante, G. (2018). The effect of HRM practices on knowledge management capacity: a comparative study in Indian IT industry. Journal of Knowledge Management, 22(3), 649-677. https://doi.org/10.1108/JKM-10-2017-0453

Greenwood, D. J. (2009). Are research universities knowledge-intensive learning organizations? In D. Jemielniak, \& J. Kociatkiewicz (Eds.), Handbook of research on knowledge-intensive organizations (p. 1-18). Hershey, PA: Information Science Reference.

Ichijo, K., \& Nonaka, I. (2006). Knowledge creation and management: New challenges for managers. Oxford university press.

Iqbal, S., Toulson, P., \& Tweed, D. (2015). Employees as performers in knowledge intensive firms: role of knowledge sharing. International Journal of Manpower, 36(7), 1072-1094. https://doi.org/10.1108/IJM-10-2013-0241

Jackson, S.E., Chuang, C.H., Harden, E.E. \& Jiang, Y. (2006). Toward developing Human Resource Management systems for knowledge-intensive teamwork. Personnel and Human Resources Management, 15, 17-70.

Juneja, J., \& Amar, A. (2018). An organizational capital decision model for knowledge-intensive organizations. IEEE Transactions on Engineering Management, 65(3), 417-433. https://doi.org/10.1109/TEM.2018.2790898

Kaiser, S., Kozica, A., Swart, J., \& Werr, A. (2015). Human resource management in professional service firms: Learning from a framework for research and practice. Zeitschrift Fur Personalforschung, 29(2), 77-101. https://doi.org/10.1688/ZfP-2015-02-Kaiser

Kamaruddin, R., Abdullah, N., \& Ayob, M. (2018). Determinants of job satisfaction among Malaysian youth working in the oil palm plantation sector. Journal of Agribusiness in Developing and Emerging Economies, 8(4), 678-692. Retrieved from http://dx.doi.org/10.1108/JADEE-01-2015$\underline{0001}$

Kemp, J., Moerman P. \& Prieto, J. (2001). On the Nature of Knowledge-intensive Organisations: Strategy and Organisation in the New Economy. 7th International Conference on Concurrent Enterprising, 251-259.

Makani, J. \& Marche, S. (2010). Towards a typology of knowledge-intensive organizations: determinant factors. Knowledge Management Research \& Practice, 8, 265-277.

Medina, R., \& Medina, A. (2017). Managing competence and learning in knowledge-intensive, project-intensive organizations: A case study of a public organization. International Journal of Managing Projects in Business, 10(3), 505-526. https://doi.org/10.1108/IJMPB-04-2016-0032

Millar, C. C. J. M., Chen, S., \& Waller, L. (2017). Leadership, knowledge and people in knowledgeintensive organisations: implications for HRM theory and practice. International Journal of Human Resource Management, 28(2), 261-275. https://doi.org/10.1080/09585192.2016.1244919 
Montoya, C., Boyero, M., y Guzmán, V. (2016). La gestión humana: un socio estratégico organizacional. Visión de Futuro, 20(1), 164-188.

Moon, G., Bong, S., \& Fardin, S. (2016). Organisational factors for effective knowledge sharing: an empirical study of Korean learning teams. International Journal of Business Innovation and Research, 10(1), 102-120. https://doi.org/10.1504/IJBIR.2016.073246

Nonaka, I., \& Takeuchi, H. (1995). The Knowledge Creating Company. Oxford: Oxford UniversityPress.

Lundvall, B. (1999). La base del conocimiento y su producción. Eknomiaz Revista Vasca de Economía, (45), 14-37.

Rivero, A., y Dabos, G. (2017). Gestión diferencial de recursos humanos: una revisión e integración de la literatura. Estudios Gerenciales, 33(142), 39-51. https://doi.org/10.1016/j.estger.2016.12.003

Schnarch, A. (2009). Desarrollo de nuevos productos y empresas: creatividad, innovación y marketing: cómo generar, desarrollar e introducir con éxito nuevos productos, servicios o empresas. (5ta ed.). Colombia: McGraw-Hill Interamericana.

Sherman, A. W., Bohlander, G. W., y Snell, S. (1999). Administración de recursos humanos. México: International Thomson Editores.

Sokeila, I., Mäkinen, S. \& Näsi, J. (2003). The Strategy Logic Of Personnel in a Knowledge-Intensive Organization. Frontiers Of E-Business Research, 393-407.

Starbuck, W. (1992).Learning by Knowledge-intensive firms. Journal of Management Studies, 29(6), 0022-2280.

Stenius, M., Haukkala, A., Hankonen, N., \& Ravaja, N. (2017). What motivates experts to share? a prospective test of the model of knowledge- sharing motivation. Human Resource Management, 56(6), 71-94. https://doi.org/10.1002/hrm

Sveiby, K. E., \& Lloyd, T. (1987). Managing knowhow. London: Bloomsbury.

Swart, J. \& Kinnie, N. (2003).Sharing Knowledge in Knowledge-intensive Firms. Human Resource Management Journal, 13(2), 60-75.

Tapscott, D. Strategy in the new economy. Strategy \& Leadership, 25(6), 8-14.

Thakur, V., \& Kumar, S. (2019). Change management for sustainability: Evaluating the role of human, operational and technological factors in leading Indian firms in home appliances sector. Journal of Cleaner Production, 213, 847-862. https://doi.org/10.1016/j.jclepro.2018.12.201 